\title{
An algorithm for rough alignment of point clouds in three- dimensional space
}

\author{
A. Makovetskii ${ }^{1}$, S. Voronin $^{1}$, V. Kober ${ }^{1}$, A. Voronin ${ }^{1}$ \\ ${ }^{1}$ Chelyabinsk State University, Bratiev Kashirinykh 129, Chelyabinsk, Russia, 454001
}

\begin{abstract}
Commonly registration of 3D point clouds consists of two steps: preliminary coarse registration and precise registration. A typical algorithm for the precise registration is the Iterative Closest Point (ICP) algorithm. To obtain an admissible result of the registration for point clouds with quite different initial positions, a coarse registration algorithm should be also be used. In this presentation, we propose a pure geometric coarse registration algorithm. For two given point clouds with arbitrary initial positions, approximation of the orthogonal transformation between the clouds is suggested. Computer simulation results are provided to illustrate the performance of the proposed algorithm.
\end{abstract}

\section{Introduction}

Point cloud registration is an important step in 3D reconstruction. Point cloud data can be obtained at different viewpoints with acquisition devices such as 3D laser scanner and Kinect camera [1]. Point cloud registration is widely used in various applications such as teleoperations, nondestructive testing, autonomous vehicle navigation, pose tracking, and SLAM [2,3]. Generally, two point clouds of an object or scene are captured at two time instances, possibly from different camera positions due to object occlusion, surface layout or measurement methods [4]. Consequently, multiple point clouds from various coordinates can be merged into a composite model with a common coordinate system.

In this paper, we propose a new fast and efficient coarse registration algorithm. The proposed algorithm consists of three steps:

1) computation of the mass centers of the template and target point clouds;

2) computation of orthogonal vector triple of the point clouds based on pure geometrical characteristics of the clouds;

3) finding the orthogonal transformation that aligns the two point clouds.

Note that proposed geometrical method is similar to the inertia ellipsoid method known in mechanics [13]. After the coarse registration, the precise alignment can be done by various ICP algorithms [14-21]. Note, that the proposed algorithm extremely improves the convergence of ICP algorithms. Computer simulation results are provided to illustrate the performance of the proposed method.

\section{Orthogonal transformation for two point clouds}

Let $P=\left\{p_{1}, \ldots, p_{s}\right\}$ be a template point cloud, and $Q=\left\{q_{1}, \ldots, q_{s}\right\}$ be a target point cloud in $\mathbb{R}^{3}$. Suppose that relationship between points in $P$ and $Q$ is given in such a manner that

$$
Q=R_{\text {true }} \mathrm{P}+t_{\text {true }},
$$

where $R_{\text {true }}$ is the rotation matrix, $t_{\text {true }}$ is the translation vector. The aim of the coarse alignment is an approximate computation of the true geometrical transformation. 


\subsection{The mass centers}

Let $c_{P}$ and $c_{Q}$ be the mass centers of the clouds $P$ and $Q$, respectively.

$$
\left\{\begin{array}{l}
c_{P}=\frac{1}{n} \sum_{i=1}^{S} p_{i} \\
c_{\mathrm{Q}}=\frac{1}{n} \sum_{i=1}^{S} q_{i}
\end{array}\right.
$$

where $c_{P}=\left(\left(c_{P}\right)_{x},\left(c_{P}\right)_{y},\left(c_{P}\right)_{z}\right)$ and $c_{Q}=\left(\left(c_{Q}\right)_{x},\left(c_{Q}\right)_{y},\left(c_{Q}\right)_{z}\right)$.

\subsection{The main axis of the point cloud}

Suppose that the main axis contains the mass center $\mathrm{c}_{\mathrm{P}}$ of the cloud $\mathrm{P}$. Denote by $v=\left(v_{x}, v_{y}, v_{z}\right)$ the directing vector of the axis. Denote the vector that connects points $c_{P}$ and $p_{i}$ by $c p_{i}$

$$
c p_{i}=\left(p_{i x}-c_{x}, p_{i y}-c_{y}, p_{i z}-c_{z}\right),
$$

and the $\mathrm{L}_{2}$ distance from the point $\mathrm{p}_{\mathrm{i}}$ to the axis by $\mathrm{d}_{\mathrm{i}}$

$$
d_{i}=\frac{\left|\left[v, c p_{i}\right]\right|}{|v|}
$$

We find the main axis of the point cloud as a straight line that is the closest to all points of the cloud in sense of the Euclidean distance in $\mathbb{R}^{3}$.

Suppose that $|v|=1$, then the following condition holds:

$$
\begin{gathered}
d_{i}=|| \begin{array}{ccc}
e_{1} & e_{2} & e_{3} \\
v_{x} & v_{y} & v_{z} \\
p_{i x}-c_{x} & p_{i y}-c_{y} & p_{i z}-c_{z}
\end{array}||= \\
=\left|\left(v_{y}\left(p_{i z}-c_{z}\right)-v_{z}\left(p_{i y}-c_{y}\right),-v_{x}\left(p_{i z}-c_{z}\right)+v_{z}\left(p_{i x}-c_{x}\right), v_{x}\left(p_{i y}-c_{y}\right)-v_{y}\left(p_{i x}-c_{x}\right)\right)\right| . \\
\text { Consider the variational problem: } \\
\sum_{i=1}^{n} \frac{\left|\left[v, c p_{i}\right]\right|^{2}}{|v|^{2}} \rightarrow \min ,
\end{gathered}
$$

subject to $|v|=1$

Denote $J(v, \lambda)$ the following functional:

$$
J(v, \lambda)=\sum_{i=1}^{n} d_{i}^{2}+\lambda\left(v_{x}^{2}+v_{y}^{2}+v_{z}^{2}-1\right),
$$

where $\lambda>0$ and consider the variational problem:

$$
J(v, \lambda) \rightarrow \min _{v, \lambda} .
$$

Note that constrained variational problem (6) is equivalent to the problem (8). Write the functional $\mathrm{J}(\mathrm{v}, \lambda)$ in the form:

$$
\begin{gathered}
J(v, \lambda)=\sum_{i=1}^{n}\left(\left(v_{y}\left(p_{i z}-c_{z}\right)-v_{z}\left(p_{i y}-c_{y}\right)\right)^{2}+\left(v_{x}\left(p_{i z}-c_{z}\right)-v_{z}\left(p_{i x}-c_{x}\right)\right)^{2}+\right. \\
+\left(v_{x}\left(p_{i y}-c_{y}\right)-v_{y}\left(p_{i x}-c_{x}\right)\right)^{2}+\lambda\left(v_{x}^{2}+v_{y}^{2}+v_{z}^{2}-1\right) .
\end{gathered}
$$

Denote by $r_{i}$ the following vector:

$$
r_{i}=\left(p_{i x}-c_{x}, p_{i y}-c_{y}, p_{i z}-c_{z}\right)
$$

Rewrite the formula (9) in the form:

$$
\begin{gathered}
J(v, \lambda)=\sum_{i=1}^{n}\left(\left(v_{y} r_{i z}-v_{z} r_{i y}\right)^{2}+\left(v_{z} r_{i x}-v_{x} r_{i z}\right)^{2}+\left(v_{x} r_{i y}-v_{y} r_{i x}\right)^{2}+\right. \\
+\lambda\left(v_{x}^{2}+v_{y}^{2}+v_{z}^{2}-1\right) .
\end{gathered}
$$

Compute the components of $\mathrm{J}(\mathrm{v}, \lambda)$ gradient

$$
\begin{aligned}
& \frac{\partial J}{\partial v_{x}}=2 v_{x}\left(\lambda+\sum_{i=1}^{n}\left({r_{i z}}^{2}+r_{i y}{ }^{2}\right)\right)-2 v_{y} \sum_{i=1}^{n}\left(r_{i x} r_{i y}\right)-2 v_{z} \sum_{i=1}^{n}\left(r_{i x} r_{i z}\right), \\
& \frac{\partial J}{\partial v_{y}}=2 v_{y}\left(\lambda+\sum_{i=1}^{n}\left(r_{i z}{ }^{2}+r_{i x}{ }^{2}\right)\right)-2 v_{x} \sum_{i=1}^{n}\left(r_{i x} r_{i y}\right)-2 v_{z} \sum_{i=1}^{n}\left(r_{i y} r_{i z}\right), \\
& \frac{\partial J}{\partial v_{z}}=2 v_{z}\left(\lambda+\sum_{i=1}^{n}\left(r_{i x}{ }^{2}+r_{i y}{ }^{2}\right)\right)-2 v_{x} \sum_{i=1}^{n}\left(r_{i x} r_{i z}\right)-2 v_{y} \sum_{i=1}^{n}\left(r_{i y} r_{i z}\right) .
\end{aligned}
$$

We obtain the following system of linear equations:

$$
\left(\begin{array}{ccc}
\lambda+\sum_{i=1}^{n}\left(r_{i z}{ }^{2}+r_{i y}{ }^{2}\right) & -\sum_{i=1}^{n}\left(r_{i x} r_{i y}\right) & -\sum_{i=1}^{n}\left(r_{i x} r_{i z}\right) \\
-\sum_{i=1}^{n}\left(r_{i x} r_{i y}\right) & \lambda+\sum_{i=1}^{n}\left(r_{i z}{ }^{2}+r_{i x}{ }^{2}\right) & -\sum_{i=1}^{n}\left(r_{i y} r_{i z}\right) \\
-\sum_{i=1}^{n}\left(r_{i x} r_{i z}\right) & -\sum_{i=1}^{n}\left(r_{i y} r_{i z}\right) & \lambda+\sum_{i=1}^{n}\left(r_{i x}{ }^{2}+r_{i y}{ }^{2}\right)
\end{array}\right)\left(\begin{array}{l}
v_{x} \\
v_{y} \\
v_{z}
\end{array}\right)=\left(\begin{array}{l}
0 \\
0 \\
0
\end{array}\right) \text {. }
$$


Rewrite the formula (14) by the expressions:

$$
\begin{gathered}
\left(\begin{array}{ccc}
\lambda+a & d & e \\
d & \lambda+b & f \\
e & f & \lambda+c
\end{array}\right)\left(\begin{array}{l}
v_{x} \\
v_{y} \\
v_{z}
\end{array}\right)=\left(\begin{array}{l}
0 \\
0 \\
0
\end{array}\right) \\
\left(\begin{array}{ccc}
-a-\lambda & -d & -e \\
-d & -b-\lambda & -f \\
-e & -f & -c-\lambda
\end{array}\right)\left(\begin{array}{l}
v_{x} \\
v_{y} \\
v_{z}
\end{array}\right)=\left(\begin{array}{l}
0 \\
0 \\
0
\end{array}\right), \\
\left.\left(\begin{array}{lll}
-a & -d & -e \\
-d & -b & -f \\
-e & -f & -c
\end{array}\right)-\lambda E\right)\left(\begin{array}{l}
v_{x} \\
v_{y} \\
v_{z}
\end{array}\right)=\left(\begin{array}{l}
0 \\
0 \\
0
\end{array}\right), \\
\left(\begin{array}{lll}
-a & -d & -e \\
-d & -b & -f \\
-e & -f & -c
\end{array}\right)\left(\begin{array}{l}
v_{x} \\
v_{y} \\
v_{z}
\end{array}\right)=\lambda\left(\begin{array}{l}
v_{x} \\
v_{y} \\
v_{z}
\end{array}\right),
\end{gathered}
$$

note that eigenvalues in the formula (19) are negative.

Denote by $A$ the matrix

$$
(-1)\left(\begin{array}{lll}
a & d & e \\
d & b & f \\
e & f & c
\end{array}\right)\left(\begin{array}{l}
v_{x} \\
v_{y} \\
v_{z}
\end{array}\right)=(-1) \lambda\left(\begin{array}{l}
a v_{x}+d v_{y}+e v_{z} \\
d v_{x}+b v_{y}+f v_{z} \\
e v_{x}+f v_{y}+c v_{z}
\end{array}\right),
$$

Then we have

$$
A=\left(\begin{array}{lll}
a & d & e \\
d & b & f \\
e & f & c
\end{array}\right)
$$

$$
\begin{gathered}
<A v, v>=\sum_{i=1}^{n}\left(\left(v_{y} r_{i z}-v_{z} r_{i y}\right)^{2}+\left(v_{z} r_{i x}-v_{x} r_{i z}\right)^{2}+\left(v_{x} r_{i y}-v_{y} r_{i x}\right)^{2}\right. \\
A v=-\lambda v \\
<-\lambda v, v>=-\lambda<v, v>=-\lambda|| v||^{2}=-\lambda= \\
=\sum_{i=1}^{n}\left(\left(v_{y} r_{i z}-v_{z} r_{i y}\right)^{2}+\left(v_{z} r_{i x}-v_{x} r_{i z}\right)^{2}+\left(v_{x} r_{i y}-v_{y} r_{i x}\right)^{2}\right.
\end{gathered}
$$

\subsection{The geometrical transformation}

We compute the mass center $c_{P}$ and orthogonal vector basis $v_{1}, v_{2}, v_{3}$ of the cloud $P$, mass center $c_{Q}$ and orthogonal vector basis $u_{1}, u_{2}, u_{3}$ of the cloud $Q$. Suppose that matrix V consists of the vectorcolumns $v_{1}, v_{2}, v_{3}$, matrix $\mathrm{U}$ consists of the vector-columns $u_{1}, u_{2}, u_{3}$. Then coarse point cloud alignment takes the following form:

$$
\begin{gathered}
t=c_{Q}-c_{P}, \\
R=\mathrm{U} V^{-1} .
\end{gathered}
$$

\section{Conclusion}

3D point cloud registration consists of two steps: preliminary coarse registration and precise registration. We proposed a pure geometric coarse registration algorithm. For two given point clouds with arbitrary initial positions, an approximation of the orthogonal transformation between the clouds is suggested.

\section{Acknowledgments}

The work was supported by the RFBR (grant № 18-07-00963).

\section{References}

[1] Zhang, Z. Microsoft Kinect Sensor and Its Effect // IEEE Multimedia. - 2012. - Vol. 19(2). - P. 4-10.

[2] Andrade-Cetto, J. Simultaneous Localization, Control and Mapping / J. Andrade-Cetto, J.A. Sanfeliu // Proc IEEE Icra05 Workshop network Robot Syst. - 2006. - Vol. 23.

[3] Engel, J. LSD-SLAM: Large-Scale DirectMonocular SLAM / J. Engel, T. Schöps, D. Cremers // Computer Vision Springer. - 2014. - Vol. 8690. - P. 834-849. 
[4] Agarwal, S. 3D point cloud registration with shape constraint / S. Agarwal, B. Bhowmick // IEEE International Conference Image Processing, 2017. - P. 2199-2203.

[5] Besl, P. Method for Registration of 3-D Shapes / P. Besl, N.A. McKay // Trans. PAMI. - 1992. - Vol. 14(2).

[6] Chen, Y. Object Modeling by Registration of Multiple Range Images / Y. Chen, G. Medioni // Proc. IEEE Conf. on Robotics and Automation, 1991.

[7] Liu, J. Three-dimensional point cloud registration based on ICP algorithmemploying K-D tree optimization // Eighth International Conference on Digital Image Processing International Society for Optics and Photonics - Cheng, China, 2016.

[8] Johnson, A. Using spin images for efficient object recognition incluttered 3D scenes / A. Johnson, M. Hebert // IEEE Transactionson Pattern Analysis \& Machine Intelligence. - 2002. Vol. 21(5). - P. 433-449.

[9] Halma, A. Singlespin Image-ICP matching for efficient 3D object recognition / A. Halma, F. Haar, E. Bovenkamp, P. Eendebak, A. Eekeren // Acm Workshop on 3d Object Retrieval ACM Firenze Italy, 2010. - P. 21-26.

[10] Lee, J. Modified Soft-Shape-Context ICP Registration System of 3-D Point Data / J. Lee, C. Huang, L. Liu, S. Lee, S. Hsieh, S. Wang // Neural Information Processing. - 2008. - Vol. 4984. - P. 723-732.

[11] Tombari, F. Unique Signatures of Histograms for Local Surface Description / F. Tombari, S. Salti, L. Stefano // Computer Vision, 11th European Conference on Computer Vision Proc, 2010. - P. 356-369. 